\title{
3 Research Square

\section{Ultra-High-Resolution CT Features of Pulmonary Nodules Correlate with Visceral Pleural Invasion in Early Stage Lung Adenocarcinoma}

Lu Li

Jinhua Central Hospital of Zhejiang University

Huimin Li

Xinhua Hospital Affiliated to Shanghai Jiaotong University School of Medicine

Jiangfeng Pan

Jinhua Central Hospital of Zhejiang University

Zhenwei Chen

Jinhua Central Hospital of Zhejiang University

Xiaorong Chen

Jinhua Central Hospital of Zhejiang University

Jiner Shu ( $\sim$ shujiner@163.com )

Jinhua people's Hospital

Peipei Pang

GE healthcare

\section{Research Article}

Keywords: Lung adenocarcinoma, visceral pleural invasion, Ultra-high-resolution CT, GGN

Posted Date: August 27th, 2021

DOI: https://doi.org/10.21203/rs.3.rs-806195/v1

License: (c) (i) This work is licensed under a Creative Commons Attribution 4.0 International License.

Read Full License 


\section{Abstract}

\section{Background}

visceral pleural invasion (VPI) is an important prognostic factor in early stage lung adenocarcinoma, which can affect the TNM Classification of Tumors.

\section{Purpose}

To investigate whether ultra-high-resolution computed tomography (U-HRCT) features can predict VPI of early stage pulmonary nodules contacting the interlobar pleura.

\section{Material and Methods}

A total of 126 patients with lung adenocarcinoma (age, 24-77 years) confirmed by surgical pathology were retrospectively enrolled. All patients underwent U-HRCT scan and were divided into two groups according to pulmonary nodular type: pure (pGGN) and mixed (mGGN). Clinical features were recorded, and U-HRCT features were manually measured using PHILIPS EBW V4.5.5. Univariate and multivariate logistic regression were used to determine factors that can significantly predict VPI.

\section{Results}

U-HRCT and three-dimensional orthogonal post-processing method could better display the relationship between GGNs and interlobar fissures. Among all patients, fifteen patients (12\%) had VPI. None of the patients with pGGN had VPI. In the mGGN group, the solid ratio (odds ratio [OR]=1.275, 95\% $\mathrm{Cl} 1.1-1.478$; $\mathrm{P}=0.001)$ and solid diameter $(\mathrm{OR}=1.139,95 \% \mathrm{Cl} 1.06-2.346 ; \mathrm{P}=0.046)$ were independent risk factors for VPI in early stage lung adenocarcinoma. For VPI diagnosis, the area under the curve, sensitivity, and specificity of the solid ratio and solid diameter were $0.803,80 \%$, and $75 \%$ and $0.807,80 \%$, and $80.36 \%$, respectively.

\section{Conclusion}

U-HRCT can display GGNs and interlobar fissures in detail. VPI was not detected in patients with pGGN. In patients with $\mathrm{mGGNs}$, a solid diameter $>6 \mathrm{~mm}$ and solid ratio $>38 \%$ can be independent predictors of VPI, which may be helpful in surgical decision-making.

\section{Introduction}

Incidence and mortality of lung cancer exhibit an increasing trend every year, and non-small cell lung cancer (NSCLC) is reported to account for about $85 \%$ of all lung cancers. Visceral pleural invasion (VPI) is an important adverse prognostic factor of NSCLC and can directly change the assessment of the clinical stage, thereby affecting the treatment strategy $(1,2)$. Oyama (3) reported 5 -year survival rates of $86 \%$, $62-70 \%$, and $57 \%$ for no pleural invasion, VPI, and parietal pleural invasion in peripheral NSCLC, 
respectively. The 8th ed of TNM staging for lung cancer, tumor with VPI is classified as T2. Some studies have suggested that a T1 sized tumor with invasion of interlobar pleura and into the adjacent lobe, it should be classified as T3, not T2, even if the tumor is $3 \mathrm{~cm}$ or less $(4,5)$. Zhao LL found that tumor size, the relationship between nodules and pleura were important predictors of VPI positivity. VPI was more common in larger $(>2 \mathrm{~cm}$ ) ground glass nodules $(G G N)$. The radiographic findings of nodule abutment or a pleural tag did not reliably predict or exclude VPI (6). However, the research to determine whether GGN of T1 lung adenocarcinoma contacting the interlobar fissure exists with VPI is lacking.

Glazer (7) proposed in 1985 that computed tomography (CT) could be used to predict pleural invasion. Different prediction and scan methods are needed based on the relationship between the tumor and pleura. VPI is more likely when the tumor is directly connected to the pleura. $\mathrm{Kim} \mathrm{H}(8)$ found that conventional CT features for pathologic VPI are not independent prognostic factors in clinical T1 lung adenocarcinomas. Thus, upstaging from clinical T1 to T2 for lung cancers closely contacting pleura or exhibiting pleural tags in CT scans is not an evidence-based practice. With the development of highresolution $\mathrm{CT}$ technology and the wide application of low-dose $\mathrm{CT}$ in lung cancer screening, the detection rate of lung GGNs is increasing. Kakinuma (9) found that ultra-high-resolution CT (U-HRCT) displayed details comparable to conventional CT but provided better image quality. In this study, we adopted the $1024 \times 1024$ matrix of the U-HRCT scanning scheme (10) combined with the three-dimensional orthogonal post-processing method, and then tried to explore the U-HRCT features as VPI prognostic factors in T1 peripheral lung adenocarcinoma in contact with the interlobar fissure, and then to help clinicians evaluate surgical options and prognosis.

\section{Material And Methods}

The Institutional Review Board of our hospital approved this retrospective study and waived the requirement for informed patient consent.

\section{Patients}

Electronic medical records were retrieved from the radiology picture archiving and communication system (PACS) in our hospital, and a retrospective analysis of clinical data, including CT findings and pathology records, from January 2014 to October 2018 was performed. Inclusion criteria were as follows: (a) lung adenocarcinoma or its preinvasive lesions equal to or less than $3 \mathrm{~cm}$ in diameter measured on chest CT imaging without distant metastases; (b) GGNs contacting the interlobar fissure; (c) surgical resection; and (d) availability of U-HRCT data with three-dimensional orthogonal post-processing (10). Excluded patients were: (a) those who had received chemotherapy, radiotherapy, or chemoradiotherapy preoperatively; (b) those with multiple primary lung cancers; (c) those without U-HRCT data. In all, 126 patients were included in the study (44 men, 82 women; mean age, $55 \pm 11$ years). Patients were divided into the pure GGN (pGGN; $n=55)$ and mixed GGN (mGGN; $n=71)$ groups.

\section{CT Scanning Protocol}


CT scanning was performed using iCT 256 Or Brilliance 64 (Philips Healthcare). All patients underwent breathing exercises before scanning, and all scans were performed under deep inspiration breath-hold. Considering the radiation dose, Only U-HRCT scan having better image quality and detail display ability than conventional CT was performed(10-12), the parameters were as follows: FOV $250 \mathrm{~mm}$, matrix $1024 \times 1024$, lateral or oblique lateral lying position with the lesion positioned as high as possible in the scanning lung field (the lesion and its surrounding background were inflated as much as possible by physiological breathing), scanning angle of view image (view angle) $90^{\circ}, 120 \mathrm{kV}, 250 \mathrm{mAs}$, reconstruction thickness $1 \mathrm{~mm}$ / space $1 \mathrm{~mm}$, FBP-D reconstruction algorithm lung window (C -500HU W 1500HU); reconstruction FOV $130-250 \mathrm{~mm}$, reconstruction thickness $0.67 \mathrm{~mm}$, space $0.3 \mathrm{~mm}$, iDose $4-6$, low-pass filtered smooth reconstruction of lung window (C -500HU W 1500HU).

\section{CT Image Processing and Evaluation}

All images were transmitted to a workstation (PHILIPS EBW V4.5.5) for measurement and threedimensional orthogonal post-processing. Multiplanar reformation (MPR) technology was carried out, and the bronchial vascular bundle or pleura was used as the reference to complete a three-dimensional orthogonal display (in our study, the correlation between GGN and interlobar pleura was studied, and the interlobar pleura was used as the reference).

MPR displayed the original layer thickness $(0.67 \mathrm{~mm}$ and $2 \mathrm{~mm})$, while volume rendering was used to display the spatial three-dimensional morphological features of the lesions. All CT images were evaluated separately by two senior chest imaging diagnostic physicians (10- and 15-years' experience) who were blinded to the pathological results. The major CT features included nodule type, size, shape, density, solid diameter, solid ratio, interface length, interface length ratio, contacting solid, pleural thickening, and pleural indentation (13-15). Maximum nodule size and maximum solid diameter were measured on a three-dimensional image. Nodule shape was divided into round or oval (partial contact with interlobar fissures), lobular (preferred when lobular characteristics were obvious), and polygonal (16). The solid ratio refers to the maximum solid diameter divided by the maximum nodule diameter (Fig. 1A). The contact length measured as curve between the tumor and the interlobar pleura was recorded as the nodule interface length. The interface length ratio was calculated by dividing the interface length by the nodule diameter (Fig. 1B). If the solid part of the nodule contacted the pleura, the length of the contact was measured as the solid interface length. For the continuous variables (solid diameter, solid ratio, et al.), the mean value measured by two doctors was used. For categorical variable (shape, Pleural indentation, et al.), the differences were resolved by a third physician (18 years' experience).

\section{Pathological Analysis}

Resected tumor and contact pleura specimens of all 126 patients were evaluated microscopically under hematoxylin-eosin (HE) staining. When the pleural elastic layer of the affected visceral layer was unclear, specific elastic fiber staining or double staining (immunohistochemical staining + elastic fiber staining) was performed. Tumor invasion degree was determined under a microscope according to modified Hammar grading criteria (1). In the 8th edition of the TNM staging system for NSCLC, VPI is defined as an 
invasion beyond the pleural elastic layer of the visceral layer (17). In our retrospectively analyzed sample, specific elastic fiber staining was performed in 33 cases and double staining was performed in 8 cases.

\section{Statistics}

All data were analyzed using SPSS 22.0 software (IBM, Armonk, NY, USA) and Medcalc statistical software (Medcalc 19, https://www.medcalc.org). The independent samples t-test or Mann-Whitney U-test was used to compare the continuous variables (represented as mean \pm standard deviation [SD]) and chisquare test or Fisher's exact test (number and percentage (\%)) to compare the categorical variables between different groups. Univariable and multivariate logistic regression (stepwise regression) was applied to identify the factors significantly correlated with VPI. Area under curve (AUC) of Receiver operating curve $(\mathrm{ROC})$ and interactive dot diagram were used to evaluate the diagnostic performance of the risk factors. A two-sided P-value of $<0.05$ was considered statistically significant.

\section{Results}

\section{Baseline characteristics of the U-HRCT findings}

U-HRCT and three-dimensional orthogonal post-processing with interlobar fissure could better display the GGN and its relationship with interlobar fissure than routine CT scan. Radiation dose from U-HRCT was quantitatively characterized by CT dose index per unit volume (CTDIvol) and Dose length product (DLP), the CTDIvol was $19.1 \pm 3.55 \mathrm{mGy}$ and DLP was $184.79 \pm 13.74 \mathrm{mGy} . \mathrm{cm}$ in the study.

A total of 126 patients meeting the inclusion criteria were included in the analysis. The baseline characteristics are shown in Table 1. The mean age was $55 \pm 11$ years, with 44 males (35\%) and 82 females $(65 \%)$, and the average nodule size was $1.4 \mathrm{~cm}$. The sample included 55 cases with pGGN and 71 cases with mGGN. Regarding the lesion type, there were 3 cases of atypical adenomatous hyperplasia (AAH), 41 cases of adenocarcinoma in situ (AIS), 26 cases of microinvasive adenocarcinoma (MIA), and 56 cases of invasive adenocarcinoma (IA). All NSCLCs with VPI were IAs, and postoperative pathology showed that none of the patients had lymph node metastasis. Morphologically, most of these NSCLCs were round or oval (70.5\%). On CT scans, most of the NSCLCs showed oblique interlobar fissure (84\%), and CT values in VPI patients were greater than those without VPI. None of the above-mentioned parameters, except age $(P=0.017)$, were significantly different $(P>0.05)$ between patients with and without VPI. 
Table 1

Baseline characteristics of the included 126 patients

\begin{tabular}{|c|c|c|c|}
\hline Clinical and imaging features & Total, n (\%) & VPI positive, $n(\%)$ & VPI negative, $\mathrm{n}(\%)$ \\
\hline Number & 126 & 15 & 111 \\
\hline Age(years) & $55 \pm 11$ & $59 \pm 13$ & $55 \pm 12$ \\
\hline \multicolumn{4}{|l|}{ Gender } \\
\hline male & $44(35 \%)$ & $4(27 \%)$ & $40(36 \%)$ \\
\hline Female & $82(65 \%)$ & $11(73 \%)$ & $71(64 \%)$ \\
\hline Nodule size (mm) & $14 \pm 6$ & $19 \pm 6$ & $13 \pm 6$ \\
\hline \multicolumn{4}{|l|}{ Nodule shape } \\
\hline round or oval & $89(70.5 \%)$ & $4(16.7 \%)$ & $85(76.6 \%)$ \\
\hline Lobulate & $12(9.5 \%)$ & $2(13.3 \%)$ & $10(9 \%)$ \\
\hline polygonal & $25(20 \%)$ & $9(60 \%)$ & $16(14.4 \%)$ \\
\hline \multicolumn{4}{|l|}{ Nodule type } \\
\hline $\mathrm{p}$ & $55(43.6 \%)$ & 0 & $55(49.5 \%)$ \\
\hline $\mathrm{m}$ & $71(56.4 \%)$ & 15 & $56(50.5 \%)$ \\
\hline \multicolumn{4}{|l|}{ Pathology } \\
\hline $\mathrm{AAH}$ & $3(2.4 \%)$ & 0 & $3(2.7 \%)$ \\
\hline AIS & $41(32.5 \%)$ & 0 & $41(37 \%)$ \\
\hline MIA & $26(20.6 \%)$ & 0 & $26(23.3 \%)$ \\
\hline IA & $56(44.5 \%)$ & $15(100 \%)$ & $41(37 \%)$ \\
\hline Lymph node metastasis & 0 & 0 & 0 \\
\hline \multicolumn{4}{|l|}{ Tumor location } \\
\hline Right upper lobe & $34(27 \%)$ & $5(33.3 \%)$ & $29(26.1 \%)$ \\
\hline Right middle lobe & $21(16.7 \%)$ & $4(26.7 \%)$ & $17(15.3 \%)$ \\
\hline Right lower lobe & $34(27 \%)$ & $4(26.7 \%)$ & $30(27 \%)$ \\
\hline Left upper lobe & $20(15.8 \%)$ & $2(13.3 \%)$ & 18(16.3\%) \\
\hline Left lower lobe & $17(13.5 \%)$ & 0 & 17(15.3\%) \\
\hline \multicolumn{4}{|l|}{ Interlobar fissures } \\
\hline Oblique fissure & $106(84 \%)$ & $11(73 \%)$ & $95(85 \%)$ \\
\hline
\end{tabular}




\begin{tabular}{|llll|}
\hline Clinical and imaging features & Total, $\mathbf{n}(\%)$ & VPI positive, $\mathbf{n}(\%)$ & VPI negative, $\mathbf{n}(\%)$ \\
\hline Split fissure & $20(16 \%)$ & $4(27 \%)$ & $16(15 \%)$ \\
\hline CT value (HU) & $-475 \pm 180$ & $-263 \pm 117$ & $-505 \pm 165$ \\
\hline
\end{tabular}

\section{U-HRCT findings of pGGN}

In the PGGN group, there were 3 cases of AAH, 35 cases of AIS, 13 cases of MIA, and 4 cases of IA. All patients with NSCLC were negative for VPI. Sixteen cases (29\%) had pleural thickening (Fig. 2), and 21 (38\%) had pleural indentation (Table 2).

Table 2

The clinical and CT findings of 55 pGGN patients

\begin{tabular}{|ll|}
\hline Imaging features & VPI negative \\
\hline Nodule size (mm) & $10 \pm 4$ \\
\hline Interface Length (mm) & $7 \pm 3$ \\
\hline Interface Length ratio (\%) & $69 \pm 19$ \\
\hline Pleural thickening & $16(29 \%)$ \\
\hline Pleural indentation & $21(38 \%)$ \\
\hline
\end{tabular}

\section{Univariate and multivariate logistic regression of $m G G N$}

The mGGN group was analyzed further as shown in Table 3. 
Table 3

The clinical and CT findings of $71 \mathrm{mGGN}$ patients

\section{Clinical and imaging} features

\section{Number}

Age

Gender

Female

male

Density

Nodule size $(\mathrm{mm})$

Tumor location

Right upper lobe

Right middle lobe

Right lower lobe

Left upper lobe

Left lower lobe

Nodule shape

round or oval

polygonal

Lobulate

\section{Pathology}

IA

AIS

MIA

Interlobar fissures

Oblique fissure

Split fissure

Pleural thickening

* $P<0.05 ; * * P<0.005$
Total VPI negative

71

71

$56.41 \pm 12.06$

$44 \quad 33$ (58.93\%)

11 (73.33\%)

1.042

0.307

$27 \quad 23(41.07 \%)$

$4(26.67 \%)$

71

$-401.96 \pm$

167.22

$-262.67 \pm$ 121.73

$-3.612$

0.001 **

$71 \quad 15.70 \pm 6.46$

$19.73 \pm 6.54$

$-2.144$

0.036 *

$19 \quad 14(25.00 \%)$

5 (33.33\%)

0.343

$10 \quad 6(10.71 \%)$

4 (26.67\%)

$22 \quad 18(32.14 \%)$

$4(26.67 \%)$

$1210(17.86 \%)$

$2(13.33 \%)$

$8 \quad 8(14.29 \%)$

$0(0.00 \%)$

\begin{tabular}{lll}
39 & $35(62.5 \%)$ & $4(26.67 \%)$ \\
\hline 21 & $12(21.43 \%)$ & $9(60.00 \%)$ \\
11 & $9(16.07 \%)$ & $2(13.33 \%)$
\end{tabular}

51

37 (66.07\%)

14 (93.33\%)

0.129

6

$6(10.71 \%)$

$0(0.00 \%)$

$14 \quad 13(23.21 \%)$

$1(6.67 \%)$

$62 \quad 51$ (91.07\%)

11 (73.33\%)

1.951

0.162

$9 \quad 5(8.93 \%)$

$4(26.67 \%)$

0.09

0.129




\begin{tabular}{|c|c|c|c|c|c|}
\hline $\begin{array}{l}\text { Clinical and imaging } \\
\text { features }\end{array}$ & Total & VPI negative & VPI positive & Statistics & P-value \\
\hline No & 23 & $20(35.71 \%)$ & $3(20.00 \%)$ & 0.713 & 0.398 \\
\hline Yes & 48 & $36(64.29 \%)$ & $12(80.00 \%)$ & & \\
\hline \multicolumn{6}{|l|}{ Pleural indentation } \\
\hline No & 19 & $18(32.14 \%)$ & $1(6.67 \%)$ & 2.726 & 0.099 \\
\hline Yes & 52 & $38(67.86 \%)$ & $14(93.33 \%)$ & & \\
\hline \multicolumn{6}{|l|}{ Contacting solid } \\
\hline No & 34 & $34(60.71 \%)$ & $0(0.00 \%)$ & 17.476 & $\begin{array}{l}<0.001 \\
\star \star\end{array}$ \\
\hline Yes & 37 & $22(39.29 \%)$ & $15(100.00 \%)$ & & \\
\hline Interface length & 71 & $9.46 \pm 5.27$ & $14.00 \pm 7.67$ & -2.157 & 0.045 * \\
\hline Solid diameter & 71 & $5.79 \pm 4.82$ & $11.73 \pm 6.94$ & -3.122 & 0.006 * \\
\hline Solid ratio & 71 & $0.35 \pm 0.17$ & $0.56 \pm 0.20$ & -3.934 & $\underset{* \star}{<0.001}$ \\
\hline Interface length ratio & 71 & $0.61 \pm 0.21$ & $0.67 \pm 0.18$ & -1.034 & 0.305 \\
\hline * $P<0.05 ; * * P<0.005$ & & & & & \\
\hline
\end{tabular}

In the mGGN group, there were 6 cases of AIS, 14 of MIA, and 51 of IA. A total of 15 (21\%) patients had VPI. The nodule sizes in patients without and with VPI were 15.7 and $19.73 \mathrm{~mm}$, the solid diameters were 5.79 and $11.73 \mathrm{~mm}$, the solid ratio was 0.35 and 0.56 , the interface length was 9.46 and $14 \mathrm{~mm}$, and the density was -401.96 and -262.67 , respectively. The nodules size, solid diameter, solid ratio and the interface length in VPI positive group all was larger than the VPI negative group, and the difference was statistically significant $(P<0.05)$. In all patients with VPI, the GGNs had solid contact with the pleura (Fig. 3 , 4). Compared to the cases without VPI, the proportion of pleural indentation $(93.33 \%$ vs. $67.86 \%)$ as well as the proportion of pleural thickening ( $80 \%$ vs. $64.29 \%)$ was higher in the cases with VPI.

Univariate and multivariate analyses were performed to identify the risk factors for pleural infiltration. Univariate analysis revealed that density, nodule size, interface length, solid diameter, and solid ratio were independent risk factors. The odds ratio (OR) values of each risk factor are shown in Table 4. After multivariate logistic regression analysis, two risk factors remained significant, namely, the solid ratio and solid diameter, with the OR values being 1.275 and 1.139 , respectively. 
Table 4

Univariate risk factors and multivariate logistic regression analysis

\begin{tabular}{|lllll|}
\hline Variable & Univariate analysis & \multicolumn{3}{l|}{ Multivariate analysis } \\
\cline { 2 - 5 } & OR $(95 \% \mathrm{Cl})$ & P-value & OR $(95 \% \mathrm{Cl})$ & P-value \\
\hline Density & $1.006(1.002,1.010)$ & 0.007 & NA & NA \\
\hline Diameter & $1.094(1.004,1.193)$ & 0.041 & NA & NA \\
\hline Contacting solid & & & NA & NA \\
\hline No & & & NA & NA \\
\hline Yes & $21434(0.0$, Inf $)$ & 0.992 & NA & NA \\
\hline Interface length & $1.117(1.021,1.223)$ & 0.016 & NA & NA \\
\hline Solid ratio & $1.166(1.059,1.283)$ & 0.002 & $1.275(1.100,1.478)$ & 0.001 \\
\hline Solid diameter & $6.582(3.424,9.157)$ & 0.001 & $1.139(1.063,2.346)$ & 0.046 \\
\hline
\end{tabular}

The areas under curve (AUC), sensitivities, and specificities of solid diameter and solid ratio for predicting VPI were $0.803,80 \%$, and $75 \%$ and $0.807,80 \%$, and $80.36 \%$, respectively (Fig. 5 and Table 5 ). There was no statistical difference between the diagnostic efficacies of the two variables by the Delong test.

Table 5

Diagnostic efficacy of independent factors

\begin{tabular}{|lllllll|}
\hline Variable & AUC $(\mathbf{9 5 \%} \mathrm{Cl})$ & Sensitivity & Specificity & Youden & Cutoff & P value \\
\hline Solid ratio & $0.803(0.69-0.89)$ & $80 \%$ & $75 \%$ & 0.55 & $>0.38$ & $<0.0001$ \\
\hline Solid diameter & $0.807(0.696-0.891)$ & $80 \%$ & $80.36 \%$ & 0.604 & $>6 \mathrm{~mm}$ & $<0.0001$ \\
\hline
\end{tabular}

\section{Discussion}

As early as 1958, Brewer recognized that VPI in lung cancer was an important prognostic factor (18), especially in early stage NSCLC. In the mid-1970s, VPI was used as a specific description in the TNM Classification of Malignant Tumors (TMN) set forth by the Union for International Cancer Control, which is now in its 8th edition (17). In the 7th edition, NSCLC with a diameter $\leq 3 \mathrm{~cm}$ and VPI was upgraded from Tla or Tlb stage to T2a stage, and the degree of VPI was classified (1). VPI was defined as tumor invasion beyond the pleural elastic layer of the visceral layer (PL1) and invasion of the pleural surface of the visceral layer (PL2). The VPI classification changes the clinician's assessment of staging and prognosis, thus affecting the treatment strategy. 
According to the TNM criteria, local invasion of a tumor in the pleura without one in the visceral layer should be classified as T2 (18). It has also been suggested that intrapulmonary pleural invasion in the interlobar region should be classified as T3 (19). Most literature reports are of T2 and T3 lung cancer, and there are few studies on T1 cases. No taxonomic study has been conducted on T1 GGN. When the tumor is in contact with the pleura, VPI is more likely to exist. As an important method to predict VPI, measuring and calculating the indicators on the corresponding CT images is highly valuable (20). Considering the radiation dose, conventional CT and U-HRCT were not performed simultaneously, only underwent U-HRCT scan were enrolled.

Based on previous research findings, compared the accuracies of U-HRCT and conventional CT for detecting morphological features on $\mathrm{CT}$ in the evaluation of GGN, The result shown that the detection rate was higher for U-HRCT target scanning than for conventional CT target reconstruction, and this improvement significantly enhanced the diagnostic accuracy of early lung adenocarcinoma (21). In UHRCT scans, a large matrix size maintained the spatial resolution and improved the image quality and assessment of lung diseases, despite an increase in image noise, when compared to a 512 matrix size, which can provide more detailed lung anatomy and pathology information for the evaluation of lung diseases $(22,23)$. Compared with the surgical pathological findings, U-HRCT had a relative higher diagnostic accuracy of early lung adenocarcinoma than conventional CT. the U-HRCT prototype scanner provides a better image quality of subsolid nodules and contributes significantly to reduce the patients' follow-up period (24). So, in this study, we adopted U-HRCT to obtain submillimeter images. Through three-dimensional orthogonal post-processing, the morphology, integrity, and features of the interlobar fissure can be displayed from different perspectives, and this information can be obtained accurately before surgery, which is more advantageous than a conventional CT scan; Total scanning dose of UHRCT is about $180 \mathrm{mGy} . \mathrm{cm}$, it was lower than that of conventional CT scan. So, we believe that HRCT will be a routine scan for further accurate diagnosis.

In this study, 17 patients (13.5\%) presented incomplete interlobar fissures, but no cases of interlobular fissures invading adjacent lobes with VPI were observed, which may be related to the low invasiveness of T1 GGN. none of the 55 pGGN contacting the pleural surface of the interlobar fissure showed VPI. The results were consistent with Hattori et al. (25), who reported that in NSCLCs with pGGN resection of less than $3 \mathrm{~cm}$, there was no VPI. Ahn et al. (9) also did not find VPI in the pGGN group. But some studies (26) show that VPI was often seen in pGGN adenocarcinoma. In our study, 4 cases of infiltrating adenocarcinoma were acini subtype, 3 had pleural thickening, 1 had pleural indentation, and 2 were cases of pathological spread through airway spaces. Pathological HE staining and elastic fiber dyeing did not reveal VPI, whereas pleural thickening and inflammatory cell infiltration was revealed in the elastic fiber layer, with no tumor cells beyond the elastic layer. All above support the fact that pGGNs are less pathologically invasive $(27,28)$ and cannot penetrate the thick elastic layer in early lung adenocarcinoma.

As for mGGN contacting the pleural surface, this study showed that there were significant differences between the cases with and without VPI in terms of density, contacting solid, solid diameter, and solid 
ratio. Some studies have confirmed the correlation between CT findings and pathological results (29), with the degree of solid component being an important invasive factor that was related to poor prognosis (30). In this study, all the positive VPI groups had solid contact with the pleura, which suggested that the solid part was an important invasive component of GGN for lung adenocarcinoma, but there was no significant difference in the interface length $(P>0.05)$. Partial solid nodules with a solid ratio greater than 0.5 were more likely to invade the visceral pleura. Ohde et al. (31) reported that in T1 NSCLC, a solid ratio greater than $50 \%$ was related to invasion, which was consistent with our study. In our study, solid diameter $>6 \mathrm{~mm}$ and solid ratio $>38 \%$ were revealed to be independent predictors of VPI $(P<0.05)$. The AUC of the two factors for VPI diagnosis reached 0.8 , and the sensitivity for both was $80 \%$, which could provide an important reference value for preoperative diagnosis of visceral pleural infiltration. In contrast to the study by Ahn et al. (13), there was no significant difference in nodule size, interface length, interface length ratio, and contacting solid in our study $(P>0.05)$, which may be related to differences in nodule types and sample size.

Previous studies on CT findings of pleural invasion have shown that pleural thickening is a useful diagnostic feature (32). Gallagher et al. (33) pointed out that there were three types of changes in the visceral pleural elastic layer: (a) no secondary changes; (b) obvious elastic overlap and inflammatory infiltration; and (c) fibroblast proliferation and thickening. The pleura is affected by inflammation and fibrosis. Even without VPI, subpleural tumors often cause anatomical changes in the pleura (1), which can manifest as pleural thickening on CT. In our study, among the 56 patients with mGGN without VPI, 36 $(64 \%)$ had pleural thickening and $38(67 \%)$ had pleural indentation. Fourteen (32\%) of 44 patients with preinvasive lesions had pleural thickening, and $19(43 \%)$ had pleural indentation. The pathology showed overlap of elastic fibers, inflammatory infiltration, and fibrosis. In our study, there was no significant difference in pleural thickening and pleural indentation in patients with mGGN with and without VPI (P values were 0.398 and 0.099 , respectively,). The results showed that pleural changes such as pleural thickening and pleural indentation were not important factors affecting VPI in NSCLC with interlobar pleural contact.

Our study has several limitations. First, our study is a retrospective study, which limits our analytical validity. Second, our study had a small sample size. Similar and larger multi-center studies are needed to confirm our current conclusions. Third, we did not evaluate inter- and intra-observer reproducibility, which may influence the CT features evaluation. The relationship between VPI and long-term outcomes such as survival, recurrence, and metastasis were not analyzed in this study. However, this study is still in progress, and relevant data, such as progression-free survival and overall survival, are in further follow-up.

In conclusion, U-HRCT and three-dimensional orthogonal post-processing technology were used to improve the spatial resolution and contrast resolution of CT images and to more accurately evaluate GGN and its relationship with interlobar fissure. This was helpful in predicting VPI. When GGN with a diameter of $\leq 3 \mathrm{~cm}$ was in contact with the interlobar fissure, the ground glass nodule component was less invasive. There was no VPI in patients with pGGN. Regarding $\mathrm{mGGN}$, a solid diameter $>6 \mathrm{~mm}$ and a solid 
ratio $>38 \%$ observed in CT evaluation could be used as an independent predictor of VPI in early stage NSCLC.

\section{Declarations}

\section{Acknowledgments}

The authors thank all the subjects who kindly agreed to participate in this study.

all methods in this study were carried out in accordance with relevant guidelines and regulations.

\section{Statement of Ethics}

This study was approved by the ethics committee of Jinhua Central Hospital of Zhejiang University; the informed consent requirement was waived.

\section{Disclosure Statement}

The authors have no conflicts of interest to declare.

\section{Funding Sources}

This work was supported by Natural Science Foundation of Zhejiang Provincial (grant number LGF18H180011) and Jinhua Science and Technology Bureau (grant number 2017-3-004, 2021-3-041).

\section{Author Contributions}

Guarantors of integrity of entire study, J.E.S, H.M.L, X.R.C; study design and data acquisition, L.L, H.M.L; manuscript drafting or revision, P.P.P: statistical analysis, Z.W.C, J.F.P; clinical studies. all authors; approval of finial version of submitted manuscript, all authors; agrees to ensure any questions related to the work are appropriately resolved.

\section{References}

1. Travis WD, Brambilla E, Rami-Porta R, et al. Visceral pleural invasion: pathologic criteria and use of elastic stains: proposal for the 7th edition of the TNM classification for lung cancer. J Thorac Oncon 2008, 3:1384-1390.

2. Jiang L, Liang W, Shen J, et al.The impact of visceral pleural invasion in node-negative non-small cell lung cancer: a systematic review and meta-analysis chest 2015, 148:903-911.

3. Mayumi Oyama, Akiko Miyagi maeshimal, Naobumi Tochigi, et al. Prognostic impact of pleural invasion 1488 patients with surgically resected non-small cell lung carcinoma. Jpn J Clin Oncol 2013, 43:540-546. 
4. Demir A, Gunluoglu MZ, Sansar D, et al. Staging and resection of lung cancer with minimal invasion of the adjacent lobe. EurJ Cardiothorac Surg 2007, 32: 855-858.

5. Ohtaki Y, Hishida T, Yoshida J, et al. The clinical outcome of non-small cell lung cancer patients with adjacent lobe invasion: the optimal classification according to the status of the interlobar pleura at the invasion point. Eur J Cardiothorac Surg 2013, 43:302-309.

6. Kim H, Goo JM, Kim YT, Park CM. CT-defined Visceral Pleural Invasion in T1 Lung Adenocarcinoma: Lack of Relationship to Disease-Free Survival. Radiology. 2019. 292(3): 741-749.

7. Glazer HS, Duncan-Meyer J, Aronberg DJ, et al. Pleural and chest wall invasion in bronchogenic carcinoma: CT evaluation. Radiology 1985, 157:191-194区

8. Zhao LL, Xie HK, Zhang LP, et al. Visceral pleural invasion in lung adenocarcinoma $\leq 3 \mathrm{~cm}$ with ground-glass opacity: a clinical, pathological and radiological study. J Thorac Dis. 2016. 8(7): 178897.

9. Kakinuma R, Moriyama N, et a1.Ultra-High-Resolution Computed Tomography of the Lung: Image Quality of a Prototype Scanner. PLoS One 2015,10: 137-145.

10. Lu Li, Hui-min Li, Jin-er Shu, et al. Influences of scan-position on clinical ultra-high-resolution CT scanning: a preliminary study. Sci Rep 2019, 9: 1134-1140.

11. Ryutaro K, Noriyuki M, Yukio M, et al. Ultra-High-Resolution Computed Tomography of the Lung: Image Quality of a Prototype Scanner. PLoS One, 2015 Sep 9,10(9):e0137165. doi:

10.1371/journal.pone.0137165. eCollection 2015.

12. Zhu $\mathrm{H}$, Zhang $\mathrm{L}$, Wang, et al. Improved image quality and diagnostic potential using ultra-highresolution computed tomography of the lung with small scan FOV: A prospective study. PLoS One, 2017,12(2):e0172688. DOI: 10.1371/journal.pone.0172688.

13. Ahn SY, Park CM, Jeon YK, et al. Predictive CT Features of Visceral Pleural Invasion by T1-Sized Peripheral Pulmonary Adenocarcinomas Manifesting as Subsolid Nodules. AJR Am J Roentgenol 2017, 209:561-566.

14. Kudo Y, Matsubayashi J, Saji H, et al. Association between high-resolution computed tomography findings and the IASLC/ATS/ERS classification of small lung adenocarcinomas in Japanese patients. Lung Cancer 2015, 90:47-54.

15. Ebara K, Takashima S, Jiang B, et al. Pleural invasion by peripheral lung cancer: prediction with threedimensional CT. Acad Radiol 2015, 22:310-319.

16. Chi Wan K, Wallace T. M, John C. K. Focal ground-glass opacities in non-small cell lung carcinoma resection patients, Eur J Radiol 2012 Jan,81(1):139-45.

17. Rami-Porta R, et al. The IASLC Lung Cancer Staging Project: Proposals for the Revisions of the T Descriptors in the Forth coming Eighth Edition of the TNM Classification for Lung Cancer. J Thorac Oncol 2015, 10: 990-1003.

18. Brewer LA, Bai AF, Little JN, et al. Carcinoma of the lung: practical classification of early diagnosis and survival treatment. JAMA 1958, 166: 1149-54. 
19. Demir A, Gunluoglu MZ, Sansar D, et al. Staging and resection of lung cancer with minimal invasion of the adjacent lobe. EurJ Cardiothorac Surg 2007, 32: 855-858.

20. Imai K, Minamiya Y, Ishiyama K, et a1. Use of CT to evaluate pleural invasion in non-small cell lung cancer冈measurement of the ratio of the interface between tumor and neighboring structures to maximum tumor diameter.Radiology 2013, 267:619-626.

21. Zhu Y, Hou D, Ma X, et al. A comparison of ultra-high-resolution CT target scan versus conventional CT target reconstruction in the evaluation of ground-glass-nodule-like lung adenocarcinoma. Quant Imaging Med Surg 2019,9(6):1087-1094

22. Akinori $H$, Masahiro $Y$, Osamu $H$, et al. Effect of Matrix Size on the Image Quality of Ultra-highresolution CT of the Lung: Comparison of $512 \times 512,1024 \times 1024$, and $2048 \times 2048$. Acad Radiol, $2018 \mathrm{Jul}, 25(7): 869-876$.

23. Tomo $M$, Masahiro $\mathrm{Y}$, Akinori $\mathrm{H}$, et al. Influence of field of view size on image quality: ultra-highresolution CT vs. conventional high-resolution CT. Eur Radiol, 2020 Jun,30(6):3324-3333.

24. Zhu $\mathrm{H}$, Zhang $\mathrm{L}$, Wang, et al. Improved image quality and diagnostic potential using ultra-highresolution computed tomography of the lung with small scan FOV: A prospective study. PLoS One, 2017,12(2): e0172688. DOI: 10.1371/journal.pone.0172688.

25. Hattori A, Suzuki K, Matsunaga T, et al. Visceral pleural invasion is not a significant prognostic factor in patients with a part-solid lung cancer. Ann Thorac Surg 2014, 98: 433-8.

26. Li-Lan Zhao, Hui-Kang Xie. et al. Visceral pleural invasion in lung adenocarcinoma $\leq 3 \mathrm{~cm}$ with ground-glass opacity: a clinical, pathological and radiological study. J Thorac Dis 2016,8: 17881797.

27. Travis, W.D., Brambilla, E., Noguchi, M. et al. International Association for the Study of Lung Cancer/American Thoracic Society/European Respiratory Society international. multidisciplinary classification of lung adenocarcinoma. J Thorac Oncol 2011, 6: 2448-5.

28. Suzuki, K, Kusumoto, M., Watanabe, S., et al. Radiologic classification of small adenocarcinoma of the lung: radiologic-pathologic correlation and its prognostic impact. Ann Thorac Surg 2006, 81: 4139.

29. Lee KH, Goo JM, Park SJ, et al. Correlation between the size of the solid component on thin-section CT and the invasive component on pathology in small lung adenocarcinomas manifesting as ground-glass nodules. J Thorac Oncol 2014, 9:74-82.

30. Naidich DP, Bankier AA, MacMahon $\mathrm{H}$, et al. Recommendations for the management of subsolid pulmonary nodules detected at CT: a statement from the Fleischner Society. Radiology 2013, 266:304-317.

31. Ohde $Y$, Nagai $K$, Yoshida J, et al. The proportion of consolidation to ground-glass opacity on high resolution $\mathrm{CT}$ is a good predictor for distinguishing the population of non-invasive peripheral adenocarcinoma. Lung Cancer 2003, 42:303-310.

32. Kuriyama K, Tateishi R, Kumatani T, et al. Pleural invasion by peripheral bronchogenic carcinoma: assessment with three-dimensional helical CT. Radiology 1994, 191:365-369. 
33. Gallagher B, Urbanski SJ. The significance of pleural elastica invasion by lung carcinomas. Hum Pathol 1990, 21:512-7.

\section{Figures}
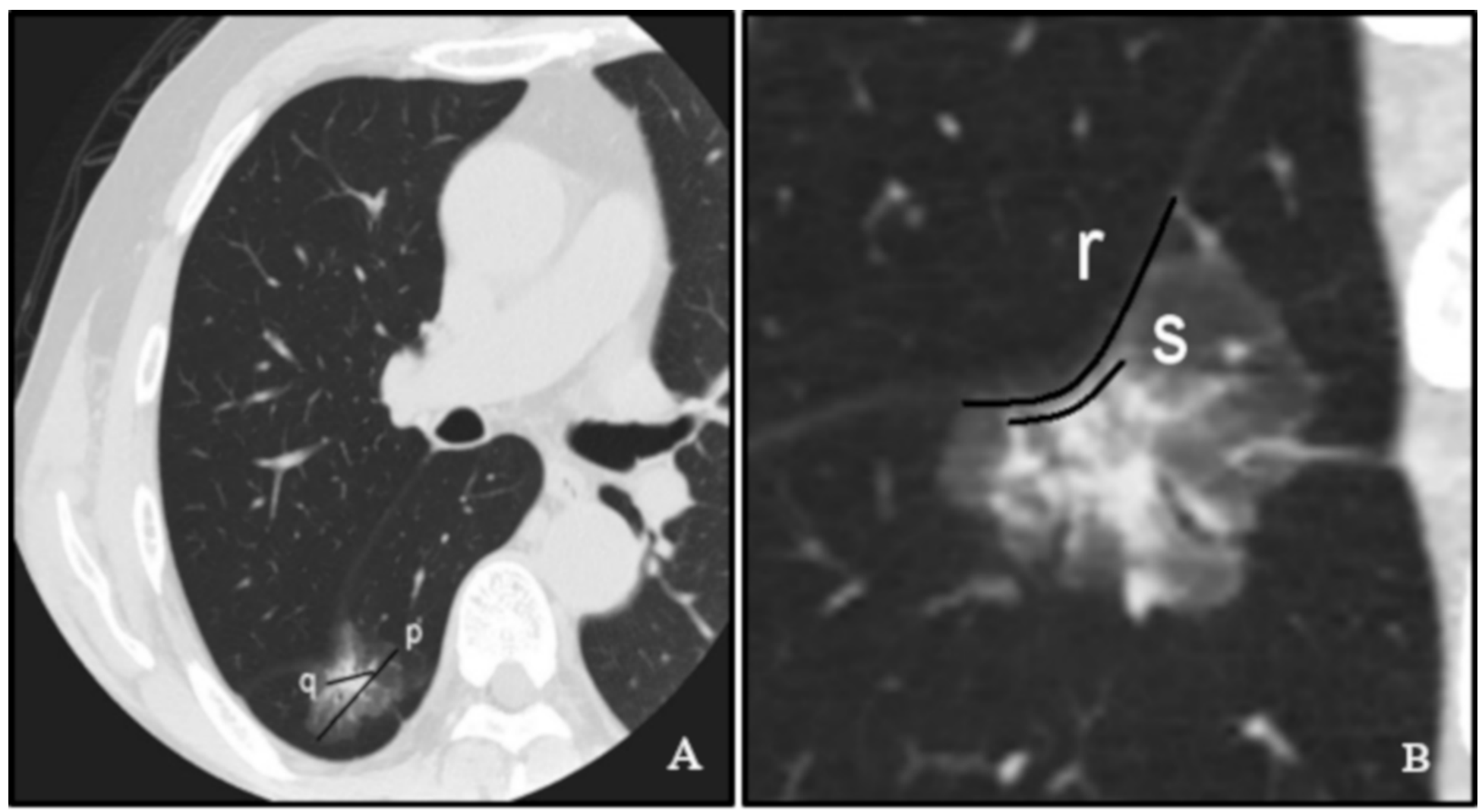

Figure 1

Parameter measurements methods. (A) Maximum diameter of ground glass nodules (GGN) (p) was measured to determine the nodule size as well as the solid diameter of mixed GGN (q) and record solid ratio $(q / p)$. (B) The interface length ( $r)$ was recorded, and the interface length ratio $(r / p)$ was calculated. If the solid part of the nodule was in contact with the interlobar pleura, the interface length was measured (s).
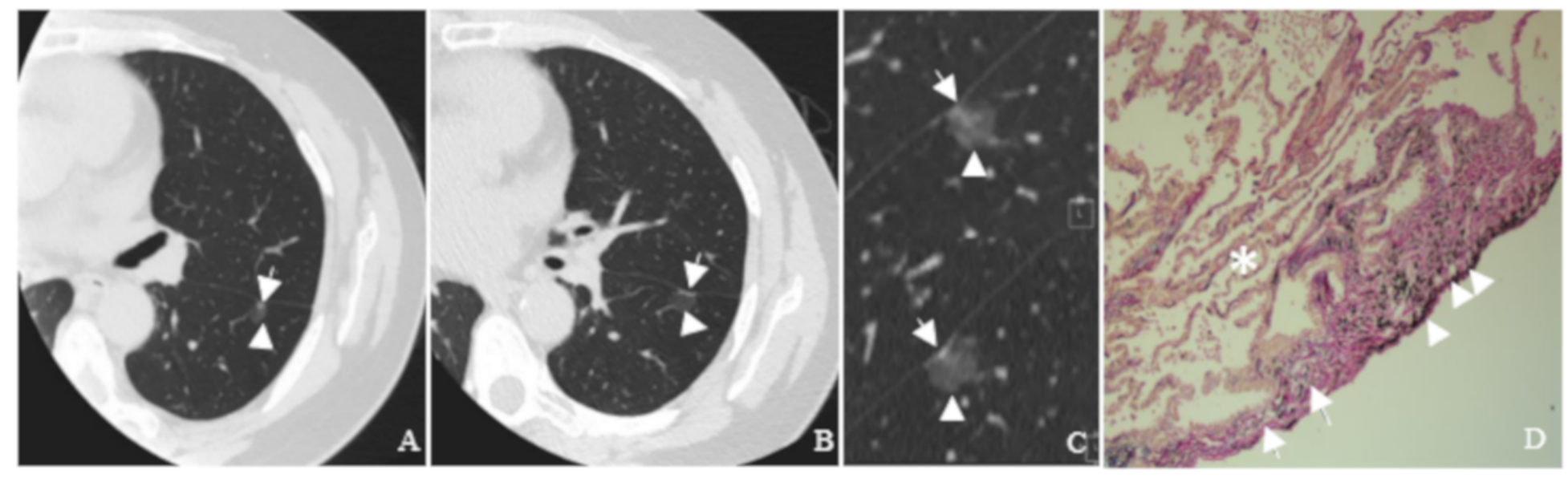


\section{Figure 2}

Computed tomography features of pure ground glass nodules (pGGN). A 61-year-old woman with alveolar adenocarcinoma without VPI. (A-C) Ultra-high-resolution computed tomography showed that in the left lower lobe, pGGN was close to the oblique fissure, and the contact surface was thickened. Lesions increased from 2016.5 (A) to 2018.5 (B) (A), and the interface length with the oblique fissure increased $(\uparrow)$. (C) Sagittal multiplanar reformation reconstruction of two scans. (D) Pathological elastic fiber stain (100X) showing a dark blue band with visceral pleural elastic layer $(\uparrow)$ and fold thickening zone multilayered elastic fibers, wherein the mixed inflammatory cells do not break the elastic layer $(\boldsymbol{\Delta})$. No. * Area of acinar adenocarcinoma.
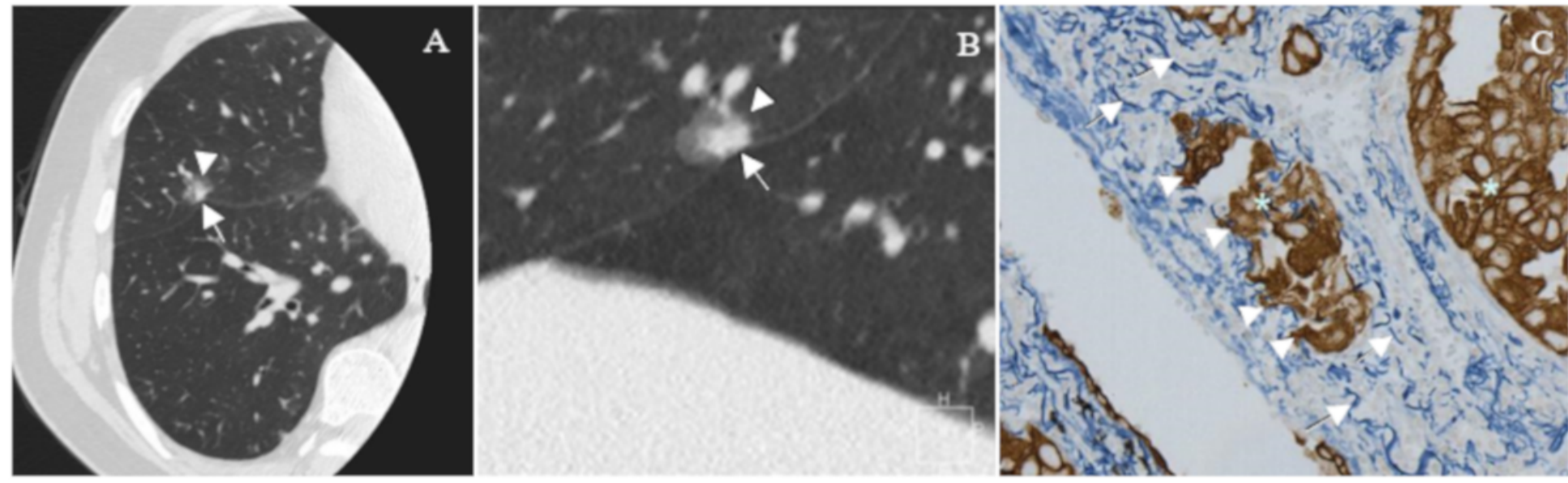

\section{Figure 3}

Example of a patient with mixed ground glass nodules (mGGN) with VPI. a 61-year-old woman with invasive adenocarcinoma with VPI. (A, B) Ultra-high-resolution computed tomography showed that in the middle lobe of the right lung, mGGN was close to the interlobar fissure ( $\mathbf{\Delta})$ : lobulate, contacting solid, pleural thickening, and indentation $(\uparrow)$, nodule size $15 \mathrm{~mm}$, solid diameter $8 \mathrm{~mm}$, solid ratio $53 \%$, and length interface ratio 40\%. (C) Pathological double staining (immunohistochemistry + elastic fiber staining [400X]) showing dark blue stripes intermittently in the visceral pleura elastic layer $(\uparrow)$ and fold thickening zone multilayered elastic fibers, wherein the mixed tumor cells $\left(^{*}\right)$ break the elastic layer $(\boldsymbol{\Delta})$ but do not reach the pleural surface.
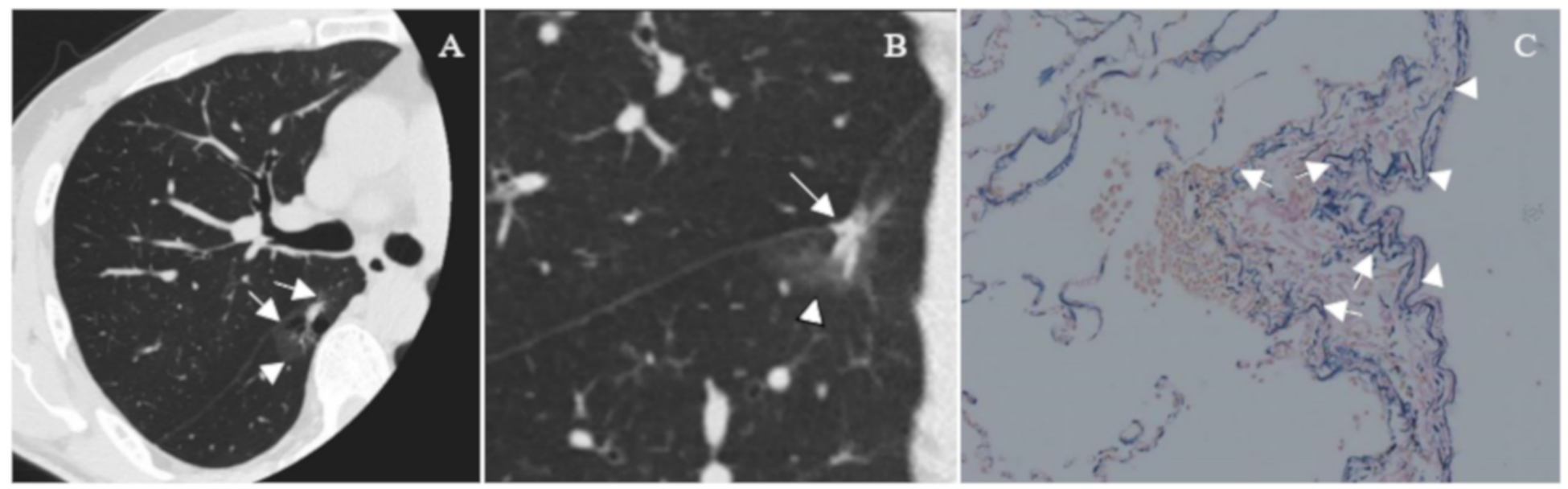


\section{Figure 4}

Example of a patient with mixed ground glass nodules (mGGN) without VPI. A 55-year-old man with invasive adenocarcinoma without VPI. Ultra-high-resolution computed tomography showed mGGN in the right lower lobe. mGGN was close to the interlobar fissure ( $\boldsymbol{\Delta})$ : semicircular, lobular, contacting solid, pleural thickening, and indentation ( $\uparrow \mathrm{A}, \mathrm{B})$, nodule size, $28 \mathrm{~mm}$, solid diameter, $6 \mathrm{~mm}$, solid ratio, $21 \%$, and length interface ratio, $89 \%$. (C) Pathological elastic fiber stain (100X). Dark blue bands in the visceral pleura elastic layer $(\uparrow)$, fold thickening zone multilayered elastic fibers, and no tumor cells breaking the elastic layer $(\boldsymbol{\Lambda})$.
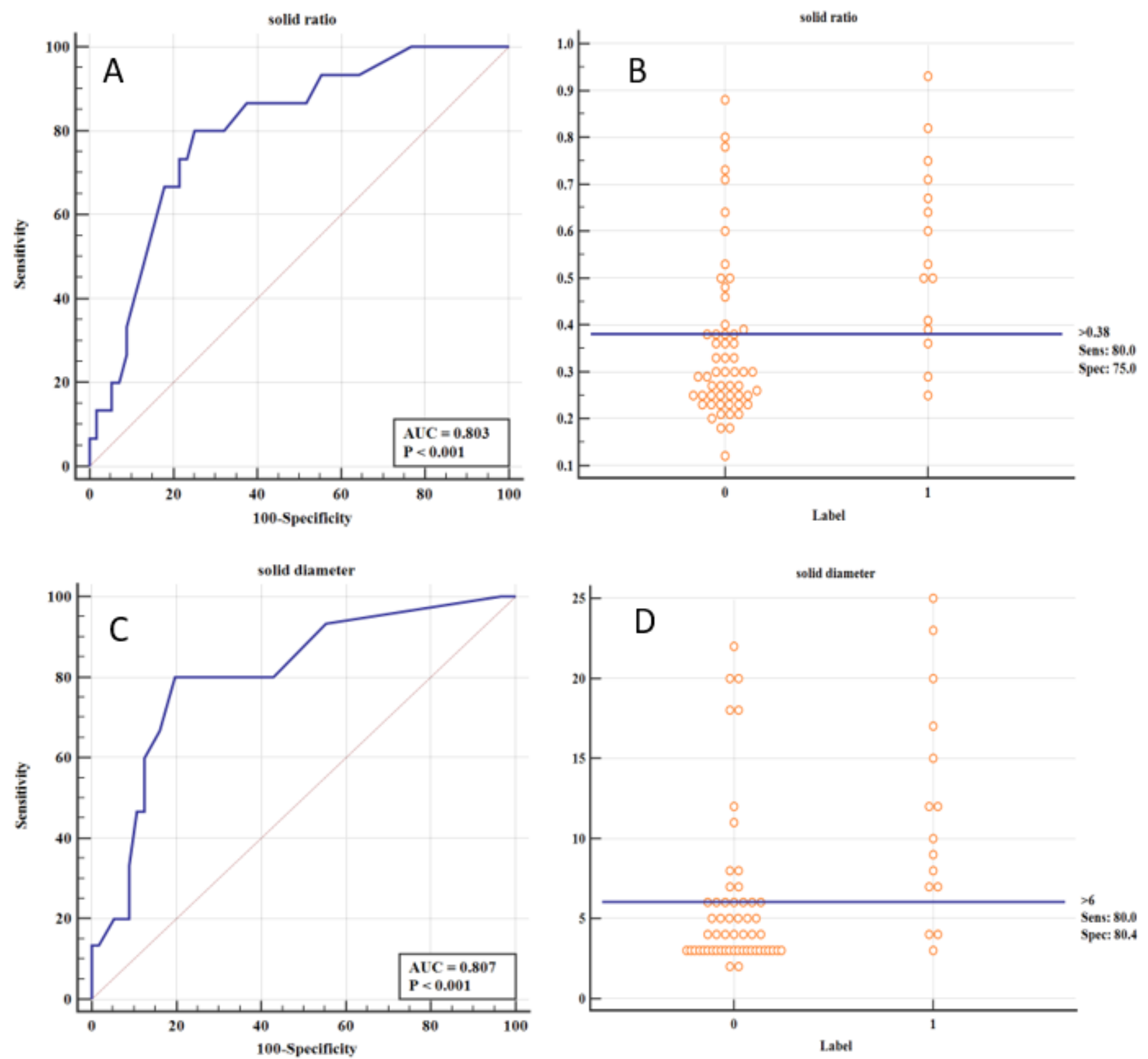

\section{Figure 5}

The diagnostic efficacy of solid diameter and solid ratio. A. ROC of solid ratio with AUC and P value, B. Interactive dot diagram of solid ratio with cutoff value, Sensitivity and Specificity, C. ROC of solid 
diameter with AUC and P value, D. Interactive dot diagram of solid diameter with cutoff value, Sensitivity and Specificity. 